Internat. J. Math. \& Math. Sci.

Vol. 24, No. 6 (2000) 379-384

S0161171200004440

(C) Hindawi Publishing Corp.

\title{
THREE-DIMENSIONAL KORTEWEG-DE VRIES EQUATION AND TRAVELING WAVE SOLUTIONS
}

\author{
KENNETH L. JONES
}

(Received 15 October 1999)

\begin{abstract}
The three-dimensional power Korteweg-de Vries equation $\left[u_{t}+u^{n} u_{x}+u_{x x x}\right]_{x}$ $+u_{y y}+u_{z z}=0$, is considered. Solitary wave solutions for any positive integer $n$ and cnoidal wave solutions for $n=1$ and $n=2$ are obtained. The cnoidal wave solutions are shown to be represented as infinite sums of solitons by using Fourier series expansions and Poisson's summation formula.
\end{abstract}

Keywords and phrases. Korteweg-de Vries equation, traveling wave solutions.

2000 Mathematics Subject Classification. Primary 35Q51, 35Q53, 76B25.

1. Introduction. In 1895, Korteweg and de Vries gave the first derivation of the nonlinear partial differential equation

$$
u_{t}+u u_{x}+u_{x x x}=0
$$

which describes the evolution of small amplitude, long water waves down a canal of rectangular cross-section [9]. This equation has been called the one-dimensional Korteweg-de Vries equation (KdV equation for short). However, the amazing properties of this equation went unappreciated for several decades until the early fifties when some related numerical work at Los Alamos produced surprising results. This led to broad interest and extensive study in this equation in the sixties and seventies. It can be found that this simplest possible unidirectional, nonlinear, and dispersive wave equation has applications in many physical problems, such as water waves, plasma waves, lattice waves, pressure waves in liquid-gas bubble mixture, and waves in elastic rods. For a survey we cite the article by Miura [10]. Once this one-dimensional equation was understood, the research for similar equations in higher dimensions and higher orders began to be studied.

In 1970, Kadomtsev and Petviashvili obtained the two-dimensional generalization of the KdV equation (referred to as KP equation henceforth)

$$
\left[u_{t}+u u_{x}+u_{x x x}\right]_{x}+u_{y y}=0
$$

in the study of plasmas [7]. The evolution described by the KP equation is weakly nonlinear, weakly dispersive, and weakly two-dimensional, with all the three effects being of the same order. 
In 1997, Bouard and Saut obtained the three-dimensional generalization of the KdV equation which can be written as the form $[5,6]$,

$$
\left[u_{t}+f(u) u_{x}+u_{x x x}\right]_{x}+u_{y y}+u_{z z}=0
$$

where $f$ is a function of $u, u=u(x, y, z, t),(x, y, z) \in \mathbb{R}^{3}$ and $t>0$. In this equation, $x$ is the direction of propagation while $y$ and $z$ are transverse variables. This model is essentially unidimensional when weak transverse effect are taken into consideration.

In this paper, the author only consider the three-dimensional KdV equation with $f(u)=u^{n}$, where $n$ is a positive integer. This equation is thus called the threedimensional power KdV equation. The author first transforms this power equation to an equivalent ordinary differential equation under no extra conditions. The solitary wave solutions for positive integer $n$ and cnoidal wave solutions when $n=1,2$, are then obtained. The author established a criterion for the existence of a single solitary wave solution, that is $C>0$, where $C=\left(a \omega-b^{2}-c^{2}\right) / a^{2}$ (see Section 3). It is also proven that the cnoidal wave solutions can be written as sums of infinite number of solitons by using Fourier series expansions and Poisson's summation formula.

2. Formulation of the problem. We start from the three-dimensional power $\mathrm{KdV}$ equation

$$
\left[u_{t}+u^{n} u_{x}+u_{x x x}\right]_{x}+u_{y y}+u_{z z}=0,
$$

where $n$ is a positive integer. Motivated by the results obtained by Chen and Wen [2], the author look for the real-valued traveling wave solutions of the form $U(\xi)=$ $u(x, y, z, t)$ with $\xi=a x+b y+c z-\omega t$, where $a, b, c$, and $\omega$ are real constants. Without loss of generality, we can assume $a>0$. Substituting the $U(\xi)$ into (2.1), we are then led to look for solutions of the following fourth order ordinary differential equation

$$
-\left(a \omega-b^{2}-c^{2}\right) U^{\prime \prime}+a^{2}\left(U^{n} U^{\prime}\right)^{\prime}+a^{4} U^{(4)}=0 .
$$

Integrating (2.2) twice with respect to $\xi$ yields the second order equation

$$
-\left(a \omega-b^{2}-c^{2}\right) U+\frac{a^{2}}{n+1} U^{n+1}+a^{4} U^{\prime \prime}=A z+B a^{2},
$$

where $A$ and $B$ are integration constants. The determination of the traveling wave solutions to the three-dimensional power $\mathrm{KdV}$ equation can now be accomplished by solving this second order differential equation.

3. Solitary wave solution. For solitary wave solutions, we introduce the boundary conditions that $U(\xi), U^{\prime}(\xi), U^{\prime \prime}(\xi), U^{\prime \prime \prime}(\xi) \rightarrow 0$ when $\xi \rightarrow \pm \infty$. These conditions imply $A=B=0$, and hence the ordinary differential equation, equation (2.3), can be written as

$$
\frac{1}{2} U^{\prime 2}=\frac{U^{2}}{a^{2}}\left[\frac{C}{2}-\frac{U^{n}}{(n+1)(n+2)}\right],
$$

where $C=\left(a \omega-b^{2}-c^{2}\right) / a^{2}$ and the author used the fact that $U^{\prime \prime}=\left(d U^{\prime 2}\right) /(2 d U)$. 
There are three cases to be considered.

First, if $C<0$, a nonconstant real solution to (3.1) exists only when $n$ is odd. The solution is

$$
U(\xi)=\left\{\frac{C(n+1)(n+2)}{2} \sec ^{2}\left[\frac{n \sqrt{-C}}{2 a}\left(\xi-\xi_{0}\right)\right]\right\}^{1 / n}
$$

where $\xi_{0}$ is a constant of integration.

Second, if $C=0$, a nonconstant real solution exists also only when $n$ is odd, and the solution has the form

$$
U(\xi)=\left\{\frac{2 a^{2}(n+1)(n+2)}{n^{2}\left(\xi-\xi_{0}\right)^{2}}\right\}^{1 / n} .
$$

It is obvious that these two solutions to (3.1) are unbounded, therefore, we are not interested in them.

Third, if $C>0$, equation (3.1) has a nontrivial solitary wave solution for positive integer $n$. The solution is

$$
U(\xi)=\left\{\frac{C(n+1)(n+2)}{2} \operatorname{sech}^{2}\left[\frac{n \sqrt{C}}{2 a}\left(\xi-\xi_{0}\right)\right]\right\}^{1 / n} .
$$

Since $\operatorname{sech}^{2} X=1 / \cosh ^{2} X=4 /\left(e^{X}+e^{-X}\right)^{2}$, the solitary waves described by the solution decay exponentially to zero when $z \rightarrow \pm \infty$. Also, since $[C(n+1)(n+2) / 2]^{1 / n}$ reduces to 1 for any given $C>0$ when $n \rightarrow \pm \infty$, the solitary waves described by the solution also become smaller and have their amplitudes reducing to 1 when $n$ increases. Furthermore, we noticed that $C>0$ gives us a condition under which a nontrivial solitary wave solution to (3.1) exists. This condition indicates that for existence of nontrivial solitary wave solutions the four constant coefficients $a, b, c$, and $\omega$, must satisfy the condition $a \omega>b^{2}+c^{2}$. On the other hand, if $a \omega \leq b^{2}+c^{2}$, either there is no real solution or the solutions are unbounded.

In particular, if we choose $b$ and $c$ relatively smaller than $\omega$ and $a, \xi_{0}=0$, and consider the weakly three-dimensional situation where $y$ and $z$ are varied in a very small region, the solitary wave solution given in (3.4) then becomes

$$
u(x, y, z, t)=U(\xi)=\left\{\frac{C(n+1)(n+2)}{2} \operatorname{sech}^{2}\left[\frac{n \sqrt{C}}{2 a}(a x-\omega t)\right]\right\}^{1 / n}
$$

4. Cnoidal wave solution when $n=1$ and $n=2$. (1) When $n=1$, for bounded periodic traveling wave solutions, we assume $A=0$, and hence, we obtain from (2.3)

$$
U^{\prime 2}=\frac{1}{3 a^{2}}\left(-U^{3}+3 C U^{2}+6 B U+D\right)=\frac{1}{3 a^{2}} F(U),
$$

where $D$ is an integration constant and $F(U)=-U^{3}+3 C U^{2}+6 B U+D$. This leads to

$$
\frac{1}{\sqrt{3} a} d z=\frac{d U}{\sqrt{F(U)}}
$$


For the existence of cnoidal wave solutions, the cubic function $F(U)$ plays an important role [8]. A cnoidal wave solution exists when $F(U)$ has three distinct real simple zeros $U_{1}, U_{2}$, and $U_{3}$ such that $U_{1}>U_{2}>U_{3}$ and $U_{2} \leq U(\xi) \leq U_{1}$. In this case (4.2) can be written as

$$
\frac{1}{\sqrt{3} a}\left(\xi-\xi_{1}\right)=\int_{U}^{U_{1}} \frac{d U}{\sqrt{\left(U_{1}-U\right)\left(U-U_{2}\right)\left(U-U_{3}\right)}},
$$

where $\xi_{1}$ is a value at which $U\left(\xi_{1}\right)=U_{1}$. The period $T$ in $\xi$ is given by

$$
T=2 \sqrt{3} a \int_{U_{2}}^{U_{1}} \frac{d U}{\sqrt{\left(U_{1}-U\right)\left(U-U_{2}\right)\left(U-U_{3}\right)}} .
$$

Equation (4.3) can also be expressed as

$$
\frac{1}{\sqrt{3} a}\left(\xi_{1}-\xi\right)=\frac{2}{\sqrt{U_{1}-U_{3}}} F(\phi, k)
$$

where $\phi=\sin ^{-1}\left[\sqrt{\left(U_{1}-U\right) /\left(U_{1}-U_{2}\right)}\right], k^{2}=\left(U_{1}-U_{2}\right) /\left(U_{1}-U_{3}\right)$, and $F(\phi, k)$ $=s n^{-1}(\sin \phi, k)$ is the normal elliptic integral of the first kind with modulus $k$ [1].

Define $v=F(\phi, k)$, we can obtain the cnoidal wave solution

$$
\begin{aligned}
U(z) & =U_{1}-\left(U_{1}-U_{2}\right) s n^{2}(v, k) \\
& =U_{2}+\left(U_{1}-U_{2}\right) c n^{2}(v, k) \\
& =U_{3}+\left(U_{1}-U_{3}\right) d n^{2}(v, k) .
\end{aligned}
$$

In particular, when $\xi_{1}=0$, if we choose $b$ and $c$ relatively smaller than $\omega$ and $a$, $\xi_{0}=0$, and consider the weakly three-dimensional situation where $y$ and $z$ are varied in a very small region, the cnoidal wave solution can be expressed as

$$
u(x, y, z, t)=U_{2}+\left(U_{1}-U_{2}\right) c n^{2}\left[\frac{1}{2 \sqrt{3} a} \sqrt{U_{1}-U_{3}}(a x-\omega t), k\right] .
$$

Using the Fourier series expansion of $d n^{2}(v, k)[11]$ and the Poisson's summation formula [4], we can represent the cnoidal wave solution $U(z)$ in (4.6) as

$$
U(\xi)=P+Q \sum_{m=-\infty}^{\infty} \operatorname{sech}^{2} R\left(\xi-\xi_{1}+m T\right),
$$

where $K=\int_{0}^{\pi / 2} d \theta / \sqrt{1-k^{2} \sin ^{2} \theta}$ is the complete elliptic integral of the first kind with modulus $k ; K^{\prime}=\int_{0}^{\pi / 2} d \theta / \sqrt{1-k^{\prime 2} \sin ^{2} \theta}$ is the complete elliptic integral of the first kind with modulus $k^{\prime}=\sqrt{1-k^{2}}$; and $E=\int_{0}^{\pi / 2} \sqrt{1-k^{2} \sin ^{2} \theta} d \theta$ is the complete elliptic integral of the second kind with modulus $k$. And

$$
\begin{gathered}
P=U_{3}+\left(U_{1}-U_{3}\right)\left[\frac{E}{K}-\frac{\pi}{2 K K^{\prime}}\right], \quad Q=\left(U_{1}-U_{3}\right) \frac{\pi^{2}}{4 K^{\prime 2}}, \\
T=\frac{4 \sqrt{3} a K}{\sqrt{U_{1}-U_{3}}}, \quad R=\frac{\pi}{2 K^{\prime} T} .
\end{gathered}
$$


In (4.8), $U(\xi)$ is clearly a periodic function of $\xi$ with period $T$ and each term in the infinite series is a soliton. This gives a representation of a periodic function by a summation of infinite number of solitons.

(2) We similarly assume $A=0$ for the case $n=2$, and obtain from (2.3),

$$
U^{\prime 2}=\frac{1}{6 a^{2}}\left(-U^{4}+6 C U^{2}+12 B U+D\right)=\frac{1}{6 a^{2}} F(U),
$$

where $F(U)=-U^{4}+6 C U^{2}+12 B U+D$.

Suppose that the constants $B, C$, and $D$ are chosen in such a way that the function $F(U)$ has four simple real zeros $U_{1}>U_{2}>U_{3}>U_{4}$ with $U_{4}=-U_{1}, U_{3}=-U_{2}$, and $U_{2} \leq U \leq U_{1}$. From (4.10) we can derive the cnoidal wave solution

$$
\begin{aligned}
U(\xi) & =\sqrt{U_{1}^{2}-\left(U_{1}^{2}-U_{2}^{2}\right) s n^{2}(v, k)} \\
& =\sqrt{U_{2}^{2}+\left(U_{1}^{2}-U_{2}^{2}\right) c n^{2}(v, k)} \\
& =U_{1} d n(v, k),
\end{aligned}
$$

where $v=-\left(U_{1} /(\sqrt{6} a)\right)\left(\xi-\xi_{1}\right), k^{2}=\left(U_{1}^{2}-U_{2}^{2}\right) / U_{1}^{2}$, and $U\left(\xi_{1}\right)=U_{1}$. This is also a periodic traveling wave solution with the period $T$ as

$$
T=2 \sqrt{6} a \int_{U_{2}}^{U_{1}} \frac{d U}{\left(U_{1}^{2}-U^{2}\right)\left(U^{2}-U_{2}^{2}\right)} .
$$

Again using the Fourier series expansion formula of $d n(v, k)$ and the Poisson's summation formula we can represent this solution as

$$
U(\xi)=Q \sum_{m=-\infty}^{\infty} \operatorname{sech} R\left(\xi-\xi_{1}+m T\right),
$$

where $Q=U_{1} \pi / 2 K^{\prime}, T=2 \sqrt{6} a K / U_{1}$, and $R=K \pi / K^{\prime} T$.

It should be mentioned that using the method used by Chen, $\mathrm{Wu}$, and Wen [3] we can also obtain the cnoidal solution for the three-dimensional power KdV equation when $n=4$.

\section{REFERENCES}

[1] P. F. Byrd and M. D. Friedman, Handbook of Elliptic Integrals for Engineers and Scientists, 2nd ed., Revised. Die Grundlehren der mathematischen Wissenschaften, Band 67, Springer-Verlag, New York, Heidelberg, 1971. MR 43\#3506. Zbl 213.16602.

[2] Y. Chen and S.-L. Wen, Traveling wave solutions to the two-dimensional Korteweg-de Vries equation, J. Math. Anal. Appl. 127 (1987), no. 1, 226-236. MR 88k:35173. Zbl 634.35066.

[3] Y. Chen, J. Wu, and S.-L. Wen, An existence theorem of periodic travelling wave solutions to the power Kadomtsev-Petviashvili equation, Arch. Mech. (Arch. Mech. Stos.) 46 (1994), no. 5, 797-804 (1995). MR 95m:35160. Zbl 821.76006.

[4] R. Courant and D. Hilbert, Methods of Mathematical Physics. Vol. I, Interscience Publishers, Inc., New York, 1953. MR 16,426a. Zbl 053.02805.

[5] A. de Bouard and J.-C. Saut, Solitary waves of generalized Kadomtsev-Petviashvili equations, Ann. Inst. H. Poincaré Anal. Non Linéaire 14 (1997), no. 2, 211-236. MR 98a:35115. Zbl 883.35103. 
[6] Symmetries and decay of the generalized Kadomtsev-Petviashvili solitary waves, SIAM J. Math. Anal. 28 (1997), no. 5, 1064-1085. MR 99c:35208. Zbl 889.35090.

[7] B. B. Kadomtsev and V. I. Petviashvili, On the stability of solitary waves in weakly dispersing media, Sov. Phys. Dokl. 15 (1970), no. 6, 539-541. Zbl 217.25004.

[8] S. Kichenassamy and P. J. Olver, Existence and nonexistence of solitary wave solutions to higher-order model evolution equations, SIAM J. Math. Anal. 23 (1992), no. 5, 1141-1166. MR 93h:35165. Zbl 755.76023.

[9] D. J. Korteweg and G. de Vries, On the change of form of long waves advancing in a rectangular canal, and on a new type of long stationary waves, Philosophical Magazine 39 (1895), 422-443.

[10] R. M. Miura, The Korteweg-de Vries equation: a survey of results, SIAM Rev. 18 (1976), no. 3, 412-459. MR 53\#8689.

[11] F. Oberhettinger, Fourier Expansions. A Collection of Formulas, Academic Press (A subsidiary of Harcourt Brace Jovanovich, Publishers), New York, London, 1973. MR 50\#5372. Zbl 265.42002.

KenNeth L. Jones: Department of MATHematics and Computer SCienCE, FAyetTeVille STATE UNIVERSity, FAYETTEVILLE, NORTH CAROLINA 28301-4298, USA

E-mail address: kjones@uncfsu.edu 


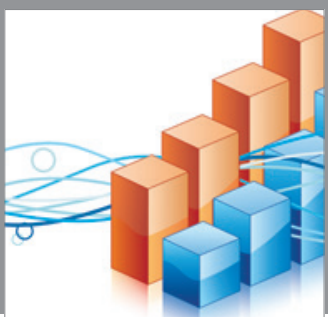

Advances in

Operations Research

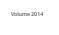

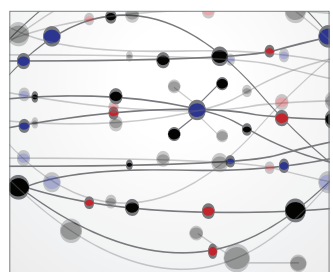

\section{The Scientific} World Journal
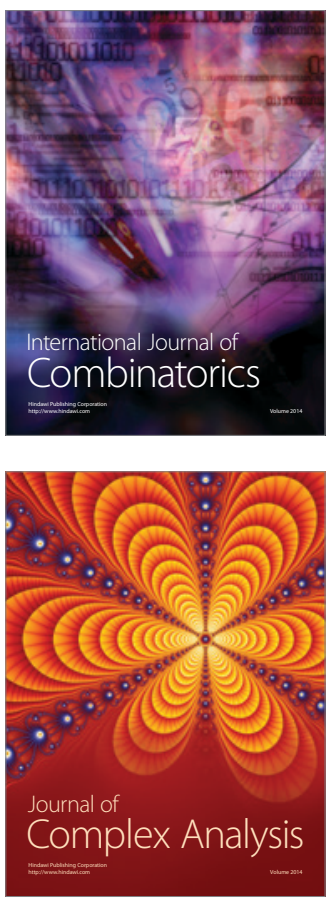

International Journal of

Mathematics and

Mathematical

Sciences
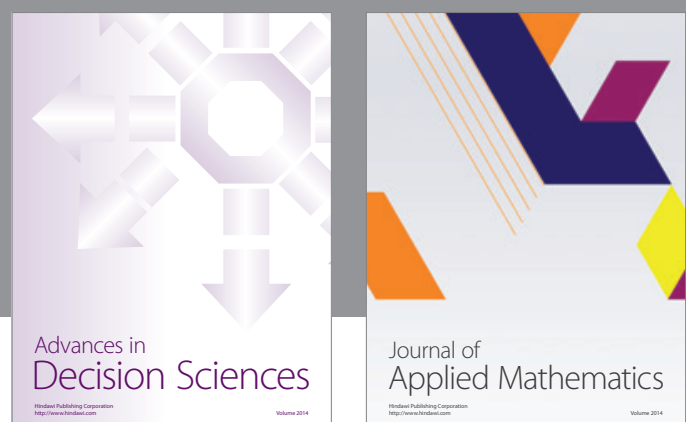

Journal of

Applied Mathematics
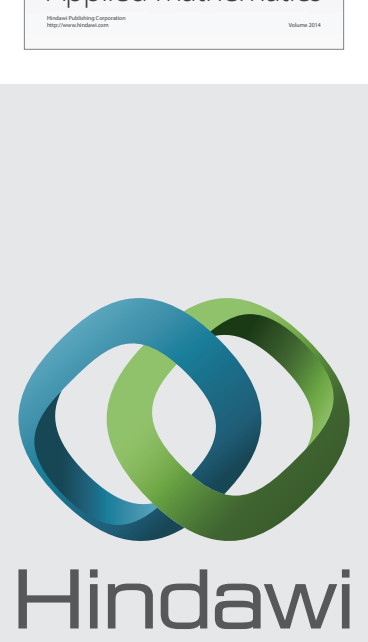

Submit your manuscripts at http://www.hindawi.com
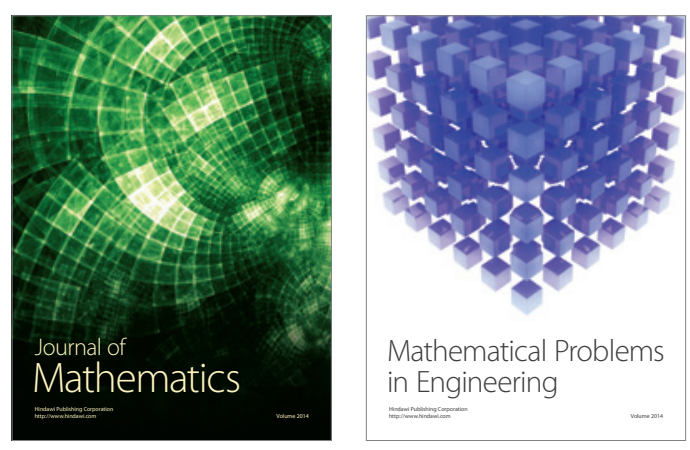

Mathematical Problems in Engineering
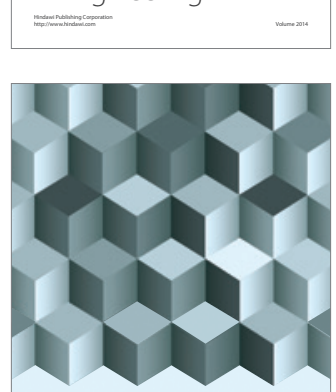

Journal of

Function Spaces
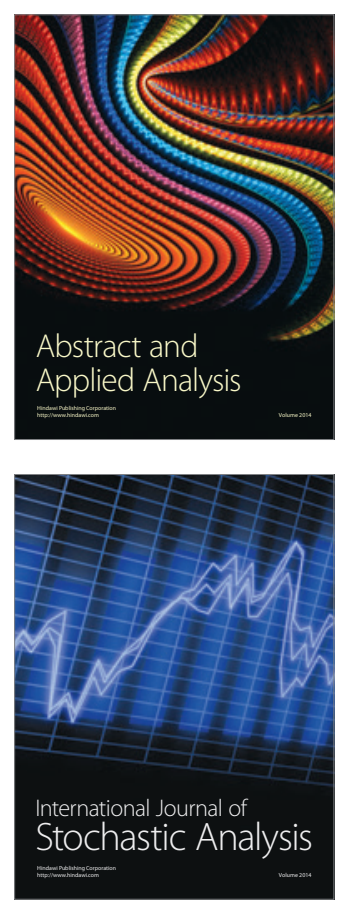

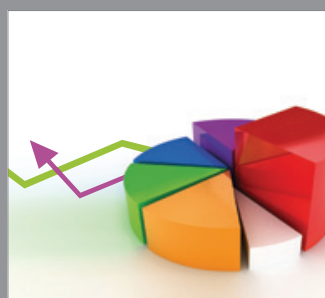

ournal of

Probability and Statistics

Promensencen
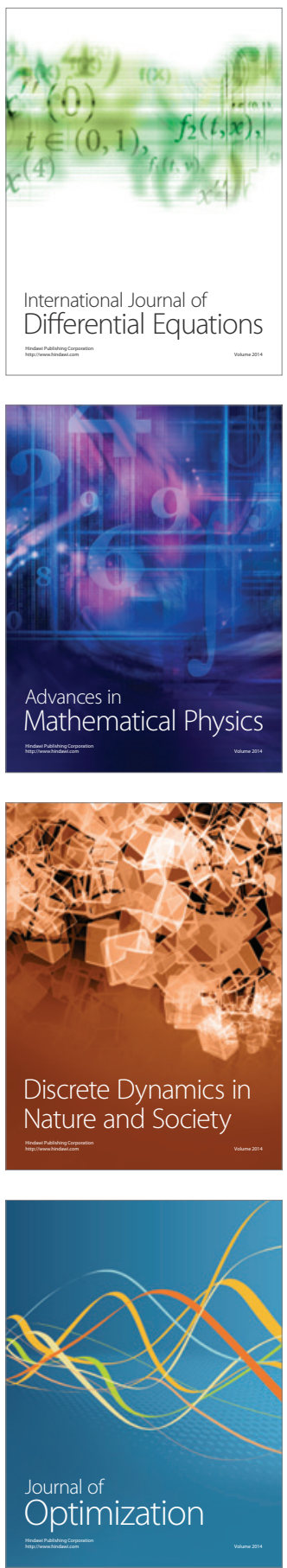